\title{
Erfolge ohne Nachhaltigkeit
}

\section{Die Jugendarbeitslosigkeit kann mit arbeitsmarktpolitischen Initiativen allein nicht bekämpft werden}

Tina Hofmann

Tina Hofmann ist Referentin für Jugendsozialarbeit beim Paritätischen Gesamtverband e. V. E-Mail jugendsozialarbeit@paritaet.org
Erfolge bei der Bekämpfung der Jugendarbeitslosigkeit wurden in den letzten Jahren auch durch arbeitsmarktpolitische Massenprogramme erzielt. Die aktuell gute Entwicklung auf dem Arbeitsmarkt sollte genutzt werden, um die Maßnahmen zukünftig individueller und passgenauer auszugestalten. Dabei muss die Verbesserung der Bildungs- und Ausbildungsvoraussetzungen von Jugendlichen ein stärkeres Gewicht bekommen. Arbeitsmarkt-und Bildungspolitik müssen dafür verknüpft werden.

Die Bekämpfung der Jugendarbeitslosigkeit steht weit oben auf der politischen Agenda der derzeitigen Bundesregierung. Sie will dabei auch die Ausbildungschancen für Jugendliche verbessern. »Möglichst alle Jugendlichen sollen eine Ausbildungschance erhalten ", so die Zielsetzung der Großen Koalition. Die Bundesregierung hat ihr Ziel, die Arbeitslosigkeit junger Menschen zu reduzieren erreicht - dank guter Konjunktur und ausgebauter Arbeitsmarktförderung.

Dennoch ist es nicht gelungen, die Jugendarbeitslosigkeit stärker als die allgemeine Arbeitslosigkeit zu senken. Die aktuelle Arbeitsmarktpolitik weist außerdem Schwächen auf, wo sie auf Massenprogramme statt auf eine individuelle Förderung der Jugendlichen setzt. In der Arbeitsmarktpolitik werden die Bildung und Qualifizierung von Jugendlichen vernachlässigt.

Arbeitsmarktpolitik für Jugendliche muss in Zukunft besser mit der Bildungspolitik verknüpft werden. Vorhandene Förderinstrumente sollten auf der lokalen Ebene koordiniert und aufeinander abgestimmt werden.

Das setzt voraus, dass Arbeitsagenturen, Träger der Grundsicherung und nicht zuletzt die Schulen mehr Handlungsspielraum erhalten.

\section{These: Nach langer Durststrecke gibt es Erfolgsmeldungen am Arbeitsmarkt auch für Jugendliche}

In ihrem Koalitionsvertrag von 2005 haben CDU und SPD angekündigt, die Jugendarbeitslosigkeit nachhaltig zu senken und die Ausbildungs- und Beschäftigungschancen von Jugendlichen zu verbessern. Unter dem Motto »Vorfahrt für junge Menschen « hat sich die Koalition darauf eingeschworen, dass in Zukunft kein Jugendlicher länger als drei Monate arbeitslos sein soll. Dabei standen die Vorzeichen zur Erreichung der ehrgeizigen Ziele in 2005 noch schlecht: Deutschland hatte über Jahre hinweg eine hohe Jugendarbeitslosigkeit zu verzeichnen. Ausgerechnet im Jahr des Amtsantritts der neuen Bundesregierung schnellte die Jugendarbeitslosigkeit um 23 Prozent nach oben und lag mit 12,5 Prozent deutlich über der ohnehin schlechten allgemeinen Arbeitslosenquote von 11,7 Prozent. Zusätzlich zu der schleppenden Konjunktur wurde die Arbeitsmarktstatistik durch den »Hartz-IV-Effekt « belastet, weil zahlreiche Jugendliche neu in der Statistik auftauchten, die zuvor nicht in Erscheinung getreten waren.

Seither hat sich der Arbeitsmarkt erstaunlich gut entwickelt und ist die Jugendarbeitslosigkeit gesunken. Die sinkenden Arbeitslosenquoten von erwerbslosen und arbeitssuchenden Jugendlichen hatten sich schon im Jahre 2006 angenähert und lagen damals bei jahresdurchschnittlich 10,8 Prozent. Im September 2007 fiel die Jugendarbeitslosigkeit jedoch erneut leicht höher aus als die allgemeine Arbeitslosigkeit (8,9\% Jugendarbeitslosigkeit, 8,4\% Arbeitslosigkeit bezogen auf alle Erwerbspersonen). Außerdem sind im Osten Deutschlands immerhin 15,6 Prozent Jugendliche arbeitslos gemeldet $(14,1 \%$ Arbeitslosenquote allgemein). Das Ziel der Bundesregierung, die Dauer der Jugendarbeitslosigkeit auf längstens drei Monate zu begrenzen, wurde knapp verfehlt; so be- 
trug die durchschnittliche Arbeitslosigkeit der jungen Menschen, die im Rechtskreis SGB II betreut wurden in 2006 vier Monate. Die Hälfte der Jugendlichen konnte allerdings ihre Arbeitslosigkeit innerhalb von nur drei Monaten beenden.

\section{These: Die Arbeitsmarktförderung setzt auf Masseneffekte statt auf maßgeschneiderte Förderung}

Die beiden Effekte - Senkung der Jugendarbeitslosigkeit und ihrer Dauer sind trotz verbesserter Konjunktur und mehr Arbeitsplätzen nur durch eine intensive Förderpolitik zu erklären. Heinrich Alt, Vorstandsmitglied der Bundesagentur für Arbeit, hat die guten Arbeitsmarktzahlen mit »steigenden Integrations- und Aktivierungsquoten, einer verbesserten Förderstruktur und dichter vernetzten Integrationsansätzen « erklärt (Pressemitteilung der Bundesagentur für Arbeit Nr. 52 vom 26. Juli 2007).

Tatsächlich nimmt ein großer Teil der arbeitslosen Jugendlichen an Fördermaßnahmen teil, mit denen die Arbeitslosenstatistik entlastet wird. Im September 2007 erhielten rund 490. 000 Jugendliche eine Förderung durch die Arbeitsagenturen und Träger der Grundsicherung - von den Arbeitsgelegenheiten über die Berufsvorbereitung bis hin zur Fortbildung. Zum gleichen Zeit- punkt waren rund 420.000 Jugendliche arbeitslos gemeldet. Der aktuellen Zielsetzung zum Trotz, die arbeitsmarktpolitischen Instrumente zu reduzieren, hat die Bundesregierung neue Förderinstrumente, darunter drei neue Kombilöhne für Jugendliche, geschaffen. Es ist fraglich, ob die massenhaft finanzierten Förderangebote immer in der erforderlichen Qualität und mit der gebotenen individuellen Ausrichtung eingesetzt werden.

So hat bei der Umsetzung des SGB II die angestrebte Verbesserung der individuellen Betreuung der Jugendlichen nicht mit dem Ausbau der Förderleistungen Schritt halten können. Der empfohlene Schlüssel von 1:75 im Fallmanagement für Jugendliche wird nicht eingehalten und liegt im Bundesdurchschnitt bei rund 1:93 (Bundestags-Drucksache 15/5837). Deshalb reicht die Personaldecke in den Arbeitsgemeinschaften und Optionskommunen häufig nicht aus, um die Jugendlichen ausreichend zu betreuen. In vielen Fällen werden immer noch keine Eingliederungsvereinbarungen mit den Jugendlichen abgeschlossen, bevor die Förderung einsetzt.

Eine passgenaue und wirksame Förderung setzt aber gerade eine intensive persönliche Beratung und Betreuung durch die persönlichen Ansprechpartner und

\section{Ein Übergangsmanagement kann klare Strukturen schaffen und steuern}

Der Kooperationsverbund Jugendsozialarbeit hat unter Federführung des Paritätischen Wohlfahrtsverbandes ein Positionspapier zur aktuellen Ausbildungsplatzsituation für benachteiligte Jugendliche erstellt. Im Mittelpunkt steht die Forderung, auch die sogenannten » nicht ausbildungsfähigen und ausbildungswilligen « Jugendlichen sollten Ausbildungschancen erhalten sollen. Ein wirksames Mittel dazu könnte die Schaffung eines »Übergangsmanagements « zwischen Schule und Beruf sein:

»Alle Angebote der beruflichen Förderung müssen kommunal und regional koordiniert werden, damit sie den Jugendlichen im Bedarfsfall überhaupt zur Verfügung stehen. Hier kommt der Kommune und ihrem Jugendamt, aber auch der freien Jugendhilfe in Zusammenarbeit mit der Agentur für Arbeit, den Jobcentern, Schulen und Kammern eine besondere Bedeutung zu. Jeweils vor Ort ist zu entscheiden, welche steuernde Instanz diese Koordinierung leisten kann, die dann mit den entsprechenden Kompetenzen und Ressourcen ausgestattet werden muss. Sie übernimmt dafür die Verantwortung, dass alle relevanten Institutionen in die Benachteiligtenförderung einbezogen werden und ein zielgerichteter Prozess organisiert wird, indem die beteiligten Institutionen und Akteure im Übergang Schule - Beruf ihre Maßnahmen und Instrumente zielgerichtet aufeinander abstimmen. Die Steuerungsinstanz stellt hierfür auch die erforderlichen Planungsdaten bereit. «

Das sechsseitige Positionspapier des Kooperationsverbundes Jugendsozialarbeit »Ausbildung für Alle - ein Ziel mit Konsequenzen für die Förderung benachteiligter Jugendlicher« kann als PDF-Datei von der Website des Paritätischen Wohlfahrtsverbandes heruntergeladen werden (http://www.paritaet.org, Rubrik Fachinformationen).

Fallmanager voraus. Notwendige Verbesserungen, die hier ansetzen müssen, sind eine bessere Personalausstattung der Arbeitsgemeinschaften und Optionskommunen, eine bessere Qualifizierung und längerfristige Beschäftigung der heute oft nur mit befristeten Arbeitsverträgen ausgestatteten Mitarbeiterinnen und Mitarbeiter.

\section{These: Die Nachhaltigkeit der}

Arbeitsmarktförderung muss erhöht werden, vor allem durch Investitionen in Bildung und Ausbildung für Jugendliche

Die Nachhaltigkeit der Arbeitsmarktpolitik für Jugendlichen muss verbessert werden, indem die Förderung auf eine Verbesserung der Ausbildungschancen von Jugendlichen gerichtet wird. Nur mit einer abgeschlossenen Berufsausbildung erhalten die Jugendlichen das Rüstzeug, um sich dauerhaft am Arbeitsmarkt behaupten zu können und nicht nur kurzfristig in prekäre Beschäftigung zu gelangen.

Bei einem Teil der Jugendlichen setzt das Ausbildungsziel voraus, dass sie zunächst einmal einen fehlenden Schulabschluss nachholen oder mit Qualifizierungsangeboten auf eine Ausbildung vorbereitet werden. Der Qualifizierungsbedarf vieler Jugendlicher, die von den Trägern der Grundsicherung betreut werden, ist enorm: Ein Viertel der arbeitslosen Jugendlichen hat keinen Schulabschluss, zwei Drittel sind ohne Berufsabschluss. Die Arbeitsgemeinschaften und Optionskommunen haben in den letzten Monaten solche Fördermaßnahmen ausgebaut, die Jugendliche auf einen Berufsabschluss vorbereiten helfen und die Ausbildungsplatzvermittlung unterstützen.

Leider schlägt sich die Qualifizierung von Jugendlichen hin zum Schul- und Berufsabschluss nicht in den Statistiken der Bundesagentur für Arbeit nieder. Sie bleibt auch bei den jährlichen Zielvereinbarungen zwischen Bundesarbeitsministerium und Bundesagentur für Arbeit zur Ausrichtung der Förderpolitik im SGB II unberücksichtigt. Ein längerfristiges und häufig kostenintensives Engagement der Arbeitsgemeinschaften und Optionskommunen für die berufliche Qualifizierung der Jugendlichen wird damit zu wenig anerkannt und zu wenig gestützt - trotz nachhaltiger Effekte auf dem Arbeitsmarkt und positiven gesellschaftlichen Nutzens. 


\section{These: Neue Initiativen für die}

\section{Ausbildung von Jugendlichen sind nötig}

Trotz der Bemühungen des Ausbildungspakts um eine Sicherung und den Ausbau betrieblicher Ausbildungsplätze hat sich im Lauf der letzten Jahre eine prekäre Situation am Ausbildungsstellenmarkt verfestigt: Förderschüler, Jugendliche mit Hauptabschluss und Jugendliche mit Migrationshintergrund haben kaum Chancen, unmittelbar nach ihrem Schulbesuch einen Ausbildungsplatz zu finden. Die Zahl der sogenannten Altbewerber ist auf einen Anteil von über 50 Prozent der Ausbildungsplatzbewerberinnen und $\mathrm{Ar}$ beitsplatzbewerber angewachsen. 15 Prozent der Jugendlichen bleiben dauerhaft ohne Berufsabschluss, bei den ausländischen Jugendlichen sogar rund 37 Prozent. Eine immer größer werdende Zahl der Jugendlichen findet erst über Umwege einen Zugang zum Ausbildungs- und Arbeitsmarkt und überbrückt Monate oder sogar Jahre in beruflichen Schulen oder Förderangeboten.

Die Bundesregierung hat den Ausbildungspakt um weitere Maßnahmen ergänzt, um diesen Jugendlichen, den Altbewerbern, gerecht $\mathrm{zu}$ werden. Die Jugendlichen erhalten mit Praktikumsangeboten eine zusätzliche Überbrückungsmöglichkeit oder werden mit Kombilöhnen in eine ungelernte Tätigkeit vermittelt. Ein sinnvoller Einsatz dieser neuen Instrumente muss mit Augenmaß erfolgen - und wird damit nicht zu den gewünschten Masseneffekten führen. Denn Kombilöhne müssen nachrangig zu ausbildungsfördernden Angeboten umgesetzt werden, damit Jugendliche ohne Berufsabschluss nicht in niedrig entlohnte Jobs abgedrängt werden.

Dabei sind die Potenziale für die Ausbildung von leistungsschwächeren Jugendlichen noch lange nicht ausgeschöpft. Der Paritätische Wohlfahrtsverband hat gemeinsam mit den im Kooperationsverbund Jugendsozialarbeit organisierten Verbänden gefordert, Zuschüsse an Betriebe auszuzahlen, die ihr Ausbildungsengagement zugunsten von Altbewerbern und leistungsschwächeren Jugendlichen steigern. Die Betriebe müssen außerdem eine begleitende Unterstützung erhalten, damit sie die Ausbildung für diese Jugendlichen organisieren können. In den westdeutschen Bundesländern werden daneben zusätzliche außerbetriebliche Ausbildungsplätze benötigt.

\section{These: Die Arbeitsmarktpolitik für Jugendliche muss mit der \\ Bildungspolitik verknüpft werden}

Arbeitsmarktpolitik darf aber nicht als Reparaturbetrieb für bildungsschwache Jugendliche überfordert werden. In erster Linie müssen die Bildungsvoraussetzungen der Jugendlichen in den Schulen verbessert werden. In der Bildungspolitik der Bundesländer muss intensiver als bisher diskutiert werden, wie berufsorientierende Angebote in den Schulen ausgebaut und dafür vorhandene Angebote der Arbeitsagenturen - etwa in der Berufsberatung - genutzt werden können. Damit die Ausbildungsförderung von Arbeitsagenturen und Trägern der Grundsicherung bei leistungsschwachen Jugendlichen frühzeitig vor dem Schulabschluss ansetzen kann, müssen die Schulen Möglichkeiten zur Kontaktaufnahme und Absprache schaffen. Arbeitsmarkt- und Bildungspolitik werden so gewinnbringend miteinander verknüpft.

\section{These: Vorhandene Förderangebote müssen besser koordiniert und aufeinander abgestimmt werden}

$\mathrm{Zu}$ den wichtigsten Herausforderungen in der zukünftigen Förderung von Jugendlichen gehört die Bündelung der Aktivitäten am Übergang von der Schule in den Beruf und die stärkere Zusammenarbeit der diversen Akteure: der allgemeinbildenden und berufsbildenden Schulen, der Unternehmen und Kammern, der Jugendämter, Trägern der Grundsicherung und Arbeitsagenturen. Die unterschiedlichen Förderinstrumente sind aufgesplittert in unterschiedliche Rechtsbereiche und Zuständigkeiten und werden in ihrer Umsetzung nur teilweise oder gar nicht aufeinander bezogen. Nötig ist deshalb ein Übergangsmanagement, mit dem die Angebote und Instrumente in den jeweiligen Regionen gesteuert und koordiniert werden. Dies muss ergänzt werden um eine verbesserte Übergangsbegleitung, die den einzelnen Jugendlichen zugute kommt.

Da die Abstimmung und Zusammenarbeit auf der örtlichen Ebene organisiert werden muss, sollten die Arbeitsagenturen mehr Handlungsspielräume erhalten, um ihre Arbeitsmarktförderung auf die lokalen Besonderheiten auszurichten. Es gilt die vorhandenen Freiräume, bei den Trägern der Grundsicherung auch zukünftig zu erhalten. Wenn der Bundesge- setzgeber in den nächsten Monaten den Versuch unternimmt, die Förderinstrumente in der Arbeitsmarktpolitik zu überprüfen und zu reduzieren, sollten die lokalen Handlungsspielräume erweitert werden: Die wirksame Förderung setzt am Individuum an und berücksichtigt die örtlichen Gegebenheiten des Wirtschaftsraums und Arbeitsmarkts. Dafür braucht es einen genügend großen Spielraum für die Handhabung der neuen Instrumente.

An die Bildungspolitik gerichtet ist die Forderung, auch den Schulen mehr Freiheitsgrade und Gestaltungsmöglichkeiten einzuräumen, damit die Schulentwicklung vorangetrieben werden kann und es den Schulen leichter gemacht wird, sich vor Ort mit der Wirtschaft, der Jugendhilfe, den Arbeitsagenturen und anderen zu vernetzen.

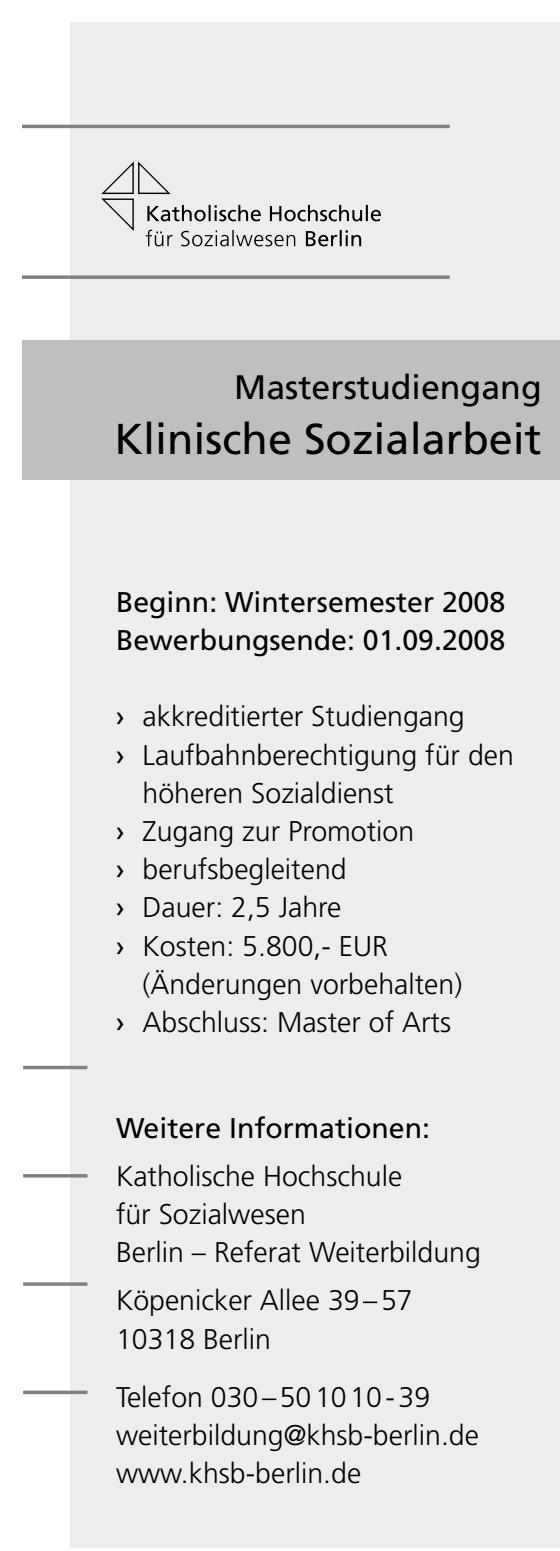

